\title{
PREVALÊNCIA DA OBESIDADE E DE HIPERTENSÃO ARTERIAL SISTÊMICA EM CRIANÇAS DE ESCOLAS PÚBLICAS DO MUNICÍPIO DE FLORIANO-PI
}

\section{PREVALENCE OF OBESITY AND SYSTEMIC ARTERIAL HYPERTENSION IN CHILDREN OF PUBLIC SCHOOLS OF FLORIANO-PI}

\author{
Alaeny Dias Clementino da Silva \\ Ada Alves da Silva ${ }^{1}$ \\ Maria Augusta Rocha Bezerra² \\ Mychelangela de Assis Brito ${ }^{2}$ \\ Ruth Cardoso Rocha ${ }^{2}$ \\ Marttem Costa de Santana ${ }^{3}$
}

\section{RESUMO}

Objetivo: Investigar a prevalência da obesidade e da hipertensão arterial sistêmica em crianças de escolas públicas do Município de Floriano-PI. Material e Métodos: Estudo descritivo, quantitativo, com delineamento transversal. Amostra composta por 349 crianças entre cinco e nove anos de idade, matriculadas em escolas municipais. Os participantes responderam a um questionário estruturado, que abrangeu dados de identificação e os antecedentes familiares dos pais e histórico de saúde. Em seguida aplicou-se um formulário, para avaliação dos dados antropométricos, níveis pressóricos e presença de Acanthosis nigricans. Realizou-se a análise estatística descritiva, desvio padrão das variáveis e teste qui-quadrado (x2). Resultados: a média de idade foi 7,8 anos, sendo $43,8 \%$ do sexo masculino e $56,2 \%$ do feminino. $24,4 \%$ das crianças apresentaram sobrepeso e obesidade; $77 \%$ tinham valores normais para pressão arterial e $15,2 \%$ estavam com valores limítrofes. 7,4\% apresentaram circunferência abdominal com percentil $\geq 90$ e $3,2 \%$ tinham Acanthosis nigricans. No que se refere ao componente genético dos pais/responsáveis foram identificados os percentuais com maior prevalência para hipertensão (45,6\%). A associação da avaliação antropométrica, nutricional, dos níveis pressóricos e da presença de Acanthosis nigricans com o sexo das crianças não se mostrou significante. Os testes qui-quadrados correlacionando o peso da criança à classificação da pressão arterial e à presença de Acanthosis nigricans apresentaram associação significativa. Conclusão: As prevalências de excesso de peso e hipertensão sistêmica foram encontradas em número reduzido de participantes, ainda assim, tornam-se um importante problema de saúde pública. Sugerem-se iniciativas que visem medidas preventivas ainda na infância.

DESCRITORES: Fatores de Risco. Criança. Doenças Cardiovasculares. Obesidade. Hipertensão.

\begin{abstract}
Objective: To investigate the prevalence of obesity and hypertension in children from public schools in the city of Floriano-PI. Methods: Descriptive study with cross-sectional design. Sample of 349 children between five and nine years old duly enrolled in public schools. We conducted a descriptive statistical analysis, standard deviation of the variables and chisquare test (X2). Results: The mean age was 7.8 years, $43.8 \%$ male and $56.2 \%$ female. $24.4 \%$ of children were overweight and obese; $77 \%$ had normal values for blood pressure (BP), $15.2 \%$ had borderline values and only $6.9 \%$ had stage 1 hypertension. $7.4 \%$ had abdominal circumference with percentile $\geq 90$ and $3.2 \%$ had Acanthosis nigricans. As regards the genetic component of parents / guardians, it was identified a greater prevalence of the percentages for hypertension (45.6\%). The association of anthropometric, nutritional, blood pressure levels and the presence of Acanthosis nigricans with the sex of the children was not significant. The chi-square tests correlating the child's weight with the classification of blood pressure and the presence of Acanthosis nigricans presented a significant association. Conclusion: The prevalence of overweight and systemic hypertension was found in a small number of participants. Even so, it has become a major public health problem. Initiatives aimed at preventive measures in childhood are suggested.
\end{abstract}

1- Enfermeira, Universidade Federal do Piauí-UFPI. Floriano, PI, Brasil.

2 - Enfermeira, Professora Assistente do Curso de Bacharelado em Enfermagem da UFPI - Campus Amílcar Ferreira Sobral, Floriano-PI. Brasil.

3- Enfermeiro, Doutorando em Tecnologia pela Universidade Tecnológica Federal do Paraná. Professor do Colégio Técnico de Floriano - Campus Amílcar Ferreira Sobral - UFPI, Floriano-PI. Brasil.

DESCRIPTORS: Risk Factors. Child. Cardiovascular diseases. Obesity. Hypertension. 
A s Doenças Cardiovasculares (DCV) são enfermidades do coração e vasos sanguíneos, incluindo variadas condições derivadas de suprimento sanguíneo diminuído a diversos órgãos do corpo ${ }^{1}$. São consideradas como uma das principais causas de morte no mundo na última década tendo início de desenvolvimento ainda nos primeiros anos de vida e quando associado aos Fatores de Risco Cardiovasculares (FRCV), pode levar ao estágio avançado da enfermidade ou à morte prematura na vida adulta ${ }^{2}$.

Dentre os fatores de risco para DCV em criança encontram-se: obesidade, hipertensão arterial sistêmica (HAS), dislipidemias, síndromes metabólicas, diabetes mellitus, sedentarismo, tabagismo passivo, sendo considerados como modificáveis. Enquanto a idade, o sexo e a história familiar encontramse dentro da categoria dos não modificáveis para doença cardiovascular ${ }^{3,4}$.

Os FRCV estão sendo identificados em crianças de forma precoce tornando-se um problema de saúde pública e estima-se que na população geral, em cada três indivíduos, um é portador de doença crônica ${ }^{5}$. Dentre estes o mais prevalente na população é a hipertensão na qual existe uma forte associação com a obesidade $^{6}$.

Recentemente, verificou-se que as prevalências para obesidade e a HAS aumentaram e variam de locais, por exemplo, a obesidade infantil chegou a atingir $41,8 \%$ no México, 22,1\% no Brasil, 22\% na Índia e 19,3\% na Argentina ${ }^{7}$. Estas prevalências diminuem para HAS, onde nos Estados Unidos e no Brasil tem sido relatados níveis de percentis de $1 \%$ a $5 \%$ e de $1 \%$ a $3 \%$ respectivamente 8,9 . A obesidade e a HAS são os FRCV que podem surgir devido ao estilo de vida sedentário, ao ambiente social em que as crianças estão inseridas e a hábitos alimentares inadequados como, por exemplo, o consumo elevado de alimentos industrializados, a alimentação fora de casa e a substituição de refeições tradicionais por fast-foods e diminuição do consumo de frutas, verduras, legumes e cereais integrais ${ }^{10}$.

Portanto, tem-se como questão de pesquisa: qual a prevalência da obesidade e da HAS em crianças no Município de Floriano-PI? A hipótese deste estudo é que, em decorrência do estilo de vida inadequado apoiado e oferecido pelos familiares, as crianças são expostas aos fatores de risco para o desencadeamento de doença cardiovascular, especificamente, a obesidade e a HAS.

Objetivou-se investigar a prevalência da obesidade e da hipertensão arterial sistêmica em crianças de escolas públicas do Município de Floriano-PI.

\section{MATERIAL E MÉTODOS}

Estudo descritivo com abordagem quantitativa e delineamento transversal, realizado em escolas municipais de FlorianoPI, entre setembro de 2014 e agosto de 2015. Segundo dados fornecidos pela Secretaria Municipal de Educação (SME), existiam no período de coleta de dados 61 estabelecimentos de ensino, com um total de 7.464 escolares matriculados entre escolas da zona urbana e rural.

Para composição da amostra, foi utilizado o cálculo de amostragem para população finita. Assim, adotando-se um 
nível de significância de $95 \%$ e um erro amostral de $5 \%$, de uma população de 3.729 crianças residentes no município na faixa etária de cinco a nove anos, conforme dados do Sistema de Informações da Atenção Básica (SIAB) referentes ao mês de setembro de 2014, obteve-se uma amostra de 349 crianças (BRASIL, 2014). Este quantitativo foi estratificado entre 10 escolas da zona urbana, sorteadas aleatoriamente, que foram incluídas como local do estudo.

Determinou-se como critérios de inclusão: estar regularmente matriculado no $1^{\circ}$ ao $9^{\circ}$ ano do ensino fundamental em uma das escolas públicas da zona urbana do Município de Floriano-PI; pais/responsáveis aceitarem responder ao questionário. Como critérios de exclusão do estudo: crianças com doenças crônicas prévias ou condições que impedissem a obtenção dos dados antropométricos e pressóricos no momento da avaliação; e a criança que não aceitasse participar do estudo, mesmo com autorização do responsável. Três crianças foram excluídas do estudo por apresentarem doenças crônicas.

A coleta de dados da pesquisa desenvolveu-se em duas etapas: a primeira envolveu a solicitação da autorização aos pais/responsáveis pelas crianças. Devido às normas e calendários das reuniões com os pais nas escolas, não foi possível realizar o encontro pessoalmente para apresentação dos objetivos da pesquisa. Desse modo, optou-se por contatar os pais por telefone. Após esse contato prévio, com a permissão dos professores apresentou-se a proposta do projeto para as crianças em sala de aula e identificou-se aquelas que aceitariam participar da pesquisa. Foi entregue o questionário e o Termo de Consentimento Livre Esclarecido (TCLE) e solicitado às crianças que levassem para suas residências e trouxessem no dia seguinte assinado pelos pais e/ou responsáveis (anteriormente contatados), autorizando a sua participação na pesquisa.

A segunda etapa envolveu os procedimentos de coleta de dados. O questionário estruturado foi aplicado com pais e responsáveis e abrangeu dados de identificação, antecedentes familiares e histórico da criança. A coleta de dados com as crianças foi realizada em uma sala reservada, na própria escola, com permissão dos professores para que os alunos se ausentassem da aula, de forma unitária. Nesta etapa, ocorreu o preenchimento do formulário com questões sobre: dados antropométricos, níveis pressóricos e presença de Acanthosis nigricans.

Para a avaliação antropométrica foi realizada a mensuração do peso, da estatura e da circunferência abdominal das crianças participantes. O peso corporal foi obtido por meio de balança digital portátil, devidamente calibrada e certificada pelo Instituto Nacional de Metrologia, Qualidade e Tecnologia (INMETRO), com capacidade máxima de 150 kg. Nessa avaliação, a criança usava roupas leves, estava descalça, com os pés permanecendo no centro da plataforma da balança. A estatura foi mensurada por meio de um estadiômetro com as crianças em posição ereta, descalças, com a cabeça na linha média e a linha na visão paralela ao teto e ao chão. O tórax posterior da criança estava contra a superfície plana vertical, com calcanhares, nádegas e ombros tocando a parede ${ }^{11}$ 
Para avaliar o estado nutricional da criança foi utilizado o IMC, sendo calculado pela equação [peso $(\mathrm{kg}) /$ altura $(\mathrm{m} 2)$ ], e avaliado conforme os gráficos de distribuição em escore $z$ do índice de massa corporal por idade para o sexo masculino e feminino. A partir desta avaliação, a criança foi classificada de acordo com estado nutricional em: magreza acentuada, magreza, eutrofia, sobrepeso, obesidade e obesidade grave ${ }^{12}$.

Foi observada a Acanthosis nigricans na pele, principalmente na região posterior do pescoço, pois a presença dessa alteração associa-se à obesidade, alterações metabólicas e a FRCV. Em seguida, esta foi avaliada e classificada conforme um instrumento em: leve, moderada e severa com as respectivas pontuações um a três, quatro a sete e oito a onze ${ }^{13}$.

A mensuração da CA foi feita utilizando uma fita métrica. Nessa avaliação, adotou-se valor médio em centímetros, passou-se pelo ponto médio, entre a borda do último arco costal e a borda da crista ilíaca anterior, na qual esta altura coincide com a linha da cicatriz umbilical. O ponto de corte para obesidade abdominal foi determinado quando a CA estava no percentil $\geq 90^{12}$.

Para aferição da PA foi utilizado o método auscultatório com utilização do estetoscópio e esfigmomanômetro devidamente calibrados, seguindo as normas recomendadas pela VI Diretrizes Brasileiras de Hipertensão ${ }^{14}$. A PA foi analisada de acordo com a faixa etária, sexo e altura, sendo considerada normal se a Pressão Sistólica (PAS) e Pressão Diastólica (PAD) fossem menores que o percentil 90 (P90).

Os instrumentos de coleta de dados foram organizados e duplamente digitados na planilha do software Microsoft Excel versão 2010 e em seguida, os dados foram exportados para software Statistical Package for Social Sciences for Windows (SPSS) (2009) versão 18.0 para geração dos resultados. Para análise dos dados foi realizada estatística descritiva, médias e desvio padrão das variáveis. O teste de qui-quadrado ( $\mathrm{x} 2$ ) foi aplicado para mostrar eventuais diferenças entre as frequências encontradas e as associações. Para todas as análises estatísticas foi considerado o Nível de Confiança de 95\% $(p<0,05)$.

A pesquisa foi aprovada pelo Comitê de Ética em Pesquisa da Universidade Federal do Piauí com o parecer de número 1.041.709, buscando atender as exigências do Conselho Nacional de Saúde no que diz respeito à execução de pesquisas com seres humanos, norteadoras pela Resolução $n^{\circ} 466 / 12$. ${ }^{15}$.

\section{RESULTADOS}

Ocorreu predominância de participantes do sexo feminino $(56,2 \%)$. A faixa etária das crianças variou de cinco a nove anos, com idade média de 7,8 anos (DP \pm $0,994)$. Prevaleceu a faixa etária de oito a nove anos, perfazendo 63,3\% (221/349).

A avaliação das variáveis antropométricas demonstrou que, quanto à estatura, a maioria das crianças encontrava-se no intervalo entre $110-\mid 125 \mathrm{~cm}$, perfazendo $57,9 \%$ da amostra (201/349). A análise inicial da variável peso envolveu quatro categorias, com o intervalo $15-\mid 30 \mathrm{~kg}$ apresentando maior prevalência - 71,9\% (251/349). Na classificação do diagnóstico nutricional obteve-se um valor mínimo 12 e máximo 34 , 
com média de 16,55 (DP $\pm 2,95)$. A partir dessa análise, identificou-se que $71,1 \%$ (248/349) das crianças apresentavam-se eutróficas, tendo uma prevalência para sobrepeso de $14,9 \%$ (52/349), para obesidade de $6,9 \%$ (24/349) e para obesidade grave de $2,6 \%$ (9/349) do total de crianças.

A circunferência abdominal apresentouse alterada, com percentil maior ou igual a 90 , em $7,4 \%$ (26/349) das crianças ${ }^{16}$. Todos os dados até aqui apresentados estão sumarizados na Tabela 1.

Em relação aos níveis pressóricos, houve um predomínio de crianças normotensas, perfazendo $93,1 \%$ (325/349) da amostra. No entanto, detectou-se que destas, 15,2\% (53/349) apresentaram valores limítrofes que indicam necessidade de acompanhamento Figura 1.

No que se refere à prevalência para Acanthosis nigricans, da amostra total, 3,2\% (11/349) crianças apresentaram esta alteração na pele. Destas, em 2,3\% (8/349) crianças a Acanthosis nigricans foi classificada como leve e em 0,9\% (3/349) participantes como moderada.

Na pesquisa, foi investigada também a história de FRCV nos antecedentes familiares

Tabela 1 - Variáveis antropométricas e análise nutricional das crianças de escolas municipais, Floriano-Piauí, 2015.

\begin{tabular}{|c|c|c|c|c|c|c|}
\hline Variáveis & $\mathrm{n}=349$ & $\%$ & Min & Max & Média & $\mathrm{Dp}$ \\
\hline \multicolumn{7}{|l|}{ Sexo da criança } \\
\hline Masculino & 153 & 43,8 & & & & \\
\hline Feminino & 196 & 56,2 & & & & \\
\hline \multicolumn{7}{|l|}{ Idade da criança } \\
\hline 5 anos & 1 & 3 & \multirow{5}{*}{5} & \multirow{5}{*}{8} & \multirow{5}{*}{7,82} & \multirow{5}{*}{ $\pm 0,994$} \\
\hline 6 anos & 38 & 10,9 & & & & \\
\hline 7 anos & 89 & 25,5 & & & & \\
\hline 8 anos & 116 & 33,2 & & & & \\
\hline$\underline{9}$ anos & 105 & 30,1 & & & & \\
\hline \multicolumn{7}{|l|}{ Estātura } \\
\hline $110-125$ & 119 & 34,1 & \multirow{3}{*}{110} & \multirow{3}{*}{155} & \multirow{3}{*}{128,83} & \multirow{3}{*}{ $\pm 8,133$} \\
\hline $125-140$ & 201 & 57,9 & & & & \\
\hline 140-145 & 29 & 8,3 & & & & \\
\hline \multicolumn{7}{|l|}{ Peso } \\
\hline $15-\mid 30$ & 251 & 71,9 & \multirow{4}{*}{15} & \multirow{4}{*}{71} & \multirow{4}{*}{27,72} & \multirow{4}{*}{ $\pm 6,924$} \\
\hline 30-45 & 87 & 24,9 & & & & \\
\hline $45-60$ & 10 & 2,9 & & & & \\
\hline$>60$ & 1 & 3 & & & & \\
\hline \multicolumn{7}{|l|}{ Diagnóstico nutricional } \\
\hline Magreza acentuada & 2 & 6 & \multirow{6}{*}{12} & \multirow{6}{*}{34} & \multirow{6}{*}{16,55} & \multirow{6}{*}{ $\pm 2,95$} \\
\hline Magreza & 14 & 4 & & & & \\
\hline Eutroficas & 248 & 71,1 & & & & \\
\hline Sobrepeso & 52 & 14,9 & & & & \\
\hline Obesidade & 24 & 6,9 & & & & \\
\hline Obesidade grave & 9 & 2,6 & & & & \\
\hline \multicolumn{7}{|c|}{ Percentil circunferência abdominal $\geq 90$} \\
\hline Sim & 26 & 7,4 & & & & \\
\hline Não & 323 & 92,6 & & & & \\
\hline
\end{tabular}


das crianças, tendo sido identificados com maiores percentuais: HAS, totalizando $45,6 \%$ (158/349); fumantes, perfazendo $41,8 \%$ (146/349); e dislipidemia, em 33,5\% (117/349) participantes na Figura 2.

Verificou-se ainda os aspectos relativos ao Aleitamento Materno Exclusivo (AME) e/ou complementar, averiguando-se que 90,3\%
(315/349) crianças haviam consumido o leite materno pelo menos em um tempo mínimo de quatro meses (Figura 3). No estudo, o tempo de AME e/ou complementar foi categorizado em duas variáveis: crianças amamentadas por quatro meses ou mais após o nascimento, que correspondeu a $66,8 \%$ (233/349) da amostra, e crianças amamentadas por um período

Figura 1 - Valores de níveis pressóricos em crianças de escolas municipais, FlorianoPiauí, 2015.

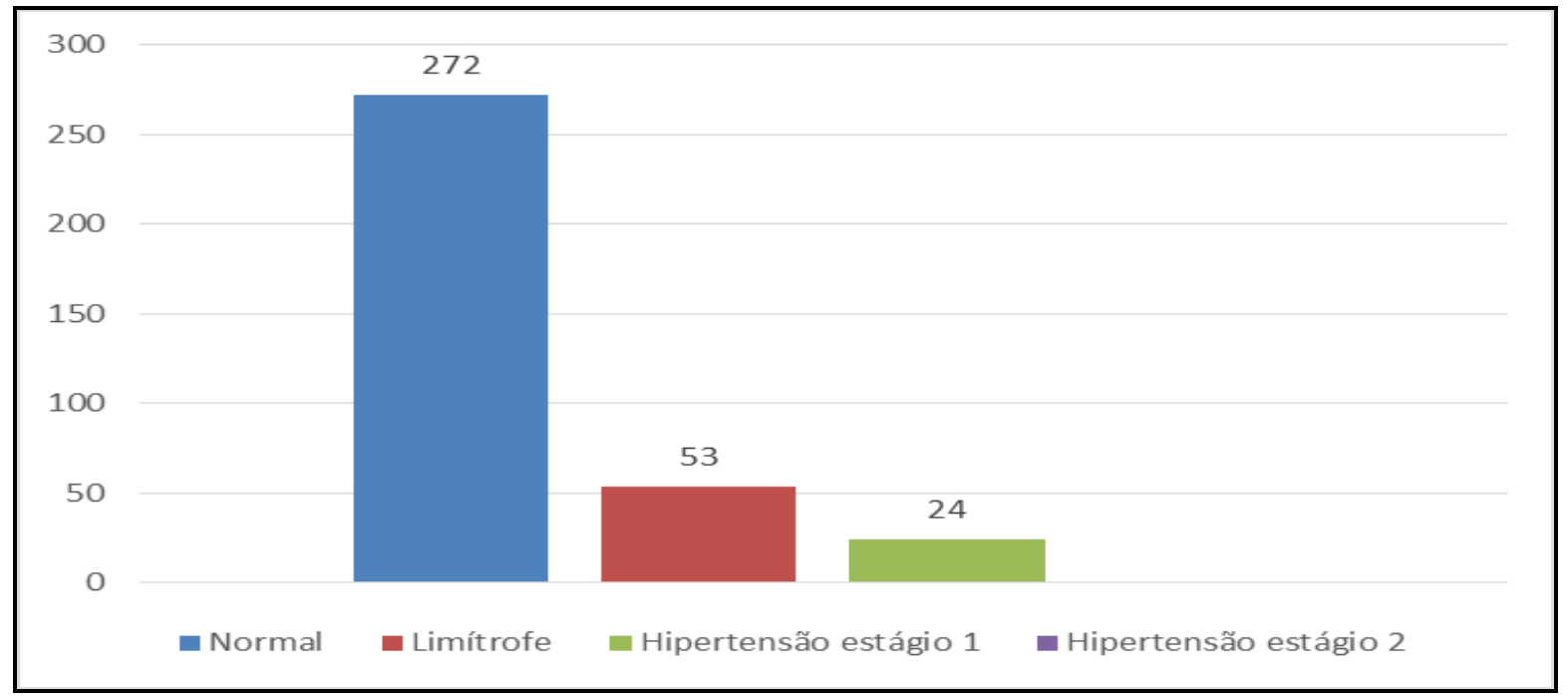

Figura 2 - Antecedentes familiares dos pais/responsáveis de crianças das escolas municipais, Floriano-Piauí, 2015.

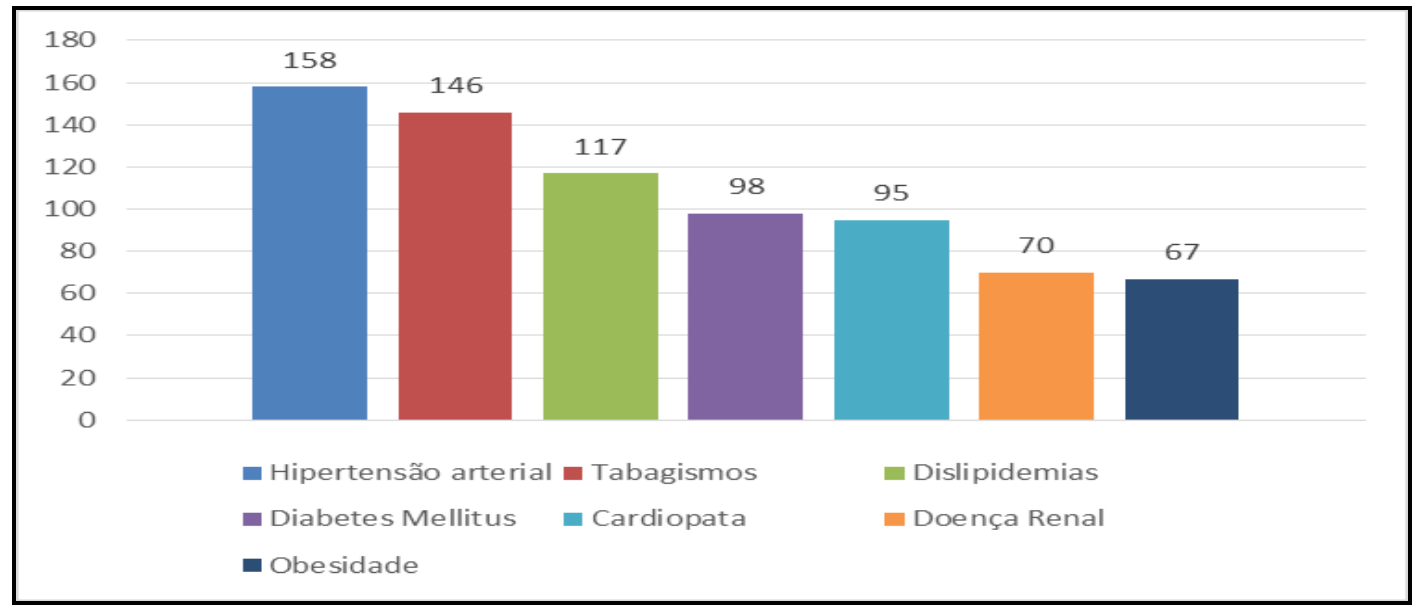


inferior a quatro meses, com 23,5\% (82/349) dos participantes, conforme estabelecido na Figura 4.

A avaliação nutricional em relação ao sexo apresentou maiores prevalências de sobrepeso e obesidade para o sexo feminino, correspondendo a 14,4\% (51/349) das escolares participantes. No outro extremo, a magreza e magreza acentuada também tiveram maior quantitativo no sexo feminino, que representou 3,3\% (12/349) da amostra na Tabela 2.

O teste de associação do qui- quadrado, apresentado na Tabela 3, para comparação da variável sexo com as variáveis antropométricas, nutricionais, com os níveis pressóricos e com a presença de Acanthosis nigricans não apresentou resultado estatisticamente significativo em nenhum dos testes considerados, sendo a significância p-valor $>0,05$.

Já os testes qui-quadrados correlacionando o peso da criança à classificação da pressão arterial e à presença de Acanthosis nigricans apresentaram associação significativa na Tabela 4.

Figura 3 - Quantidade de crianças que receberam aleitamento materno exclusivo e/ou complementar em Floriano-PI, 2015.

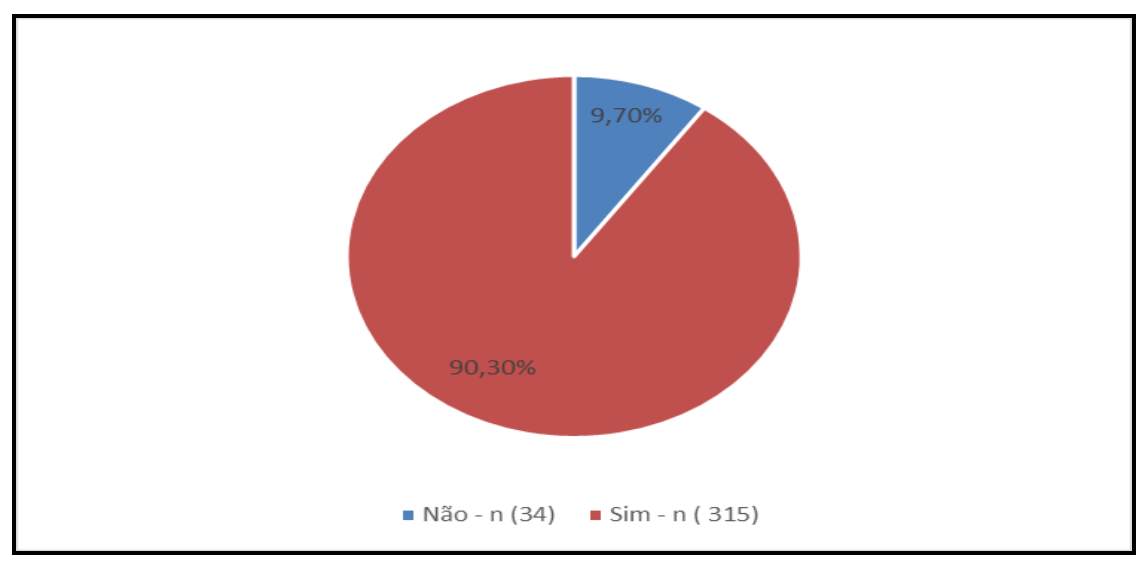

Figura 4 - Tempo de duração de aleitamento materno exclusivo e/ou complementar em Floriano-PI, 2015.

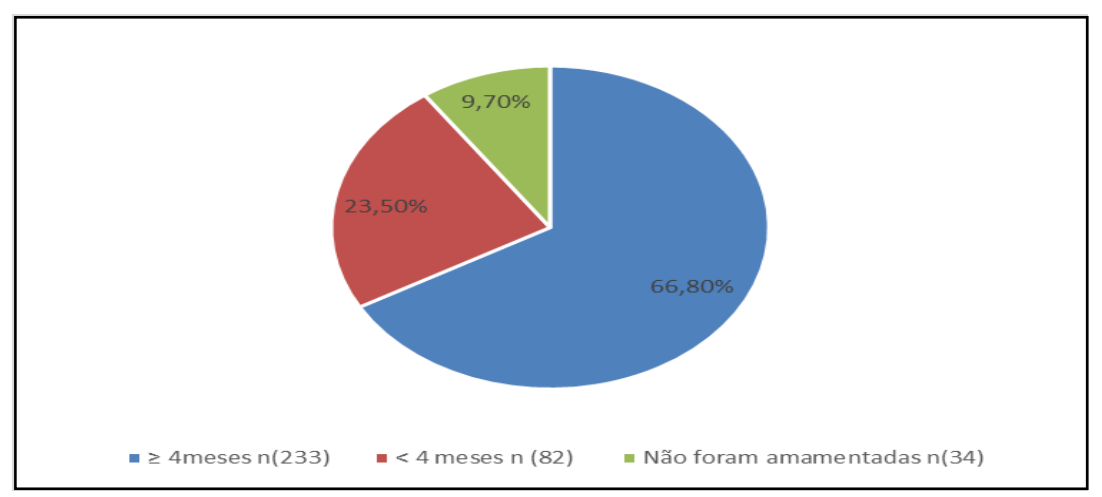


Tabela 2 - Avaliação nutricional em relação ao sexo das crianças participantes do estudo em Floriano-PI, 2015.

\begin{tabular}{cccccc}
\hline Variáveis & \multicolumn{3}{c}{ Masculino } & \multicolumn{3}{c}{ Feminino } & Total \\
& $\mathrm{n}=153$ & $\%$ & $\mathrm{n}=196$ & $\%$ & \\
\hline Diagnóstico nutricional & & & & & \\
Magreza acentuada & 0 & $0 \%$ & 2 & $0,5 \%$ & 2 \\
Magreza & 4 & $1,1 \%$ & 10 & $2,8 \%$ & 14 \\
Eutroficas & 115 & $32,9 \%$ & 133 & $38,1 \%$ & 248 \\
Sobrepeso & 19 & $5,4 \%$ & 33 & $9,4 \%$ & 52 \\
Obesidade & 9 & $2,5 \%$ & 15 & $4,2 \%$ & 24 \\
Obesidade grave & 6 & $1,7 \%$ & 3 & $0,8 \%$ & 9 \\
\hline
\end{tabular}

O teste de associação do qui-quadrado, apresentado na Tabela 3, para comparação da variável sexo com as variáveis antropométricas, nutricionais, com os níveis pressóricos e com a presença de Acanthosis nigricans não apresentou resultado estatisticamente significativo em nenhum dos testes considerados, sendo a significância pvalor $>0,05$.

Tabela 3 - Associação da avaliação antropométrica, nutricional, dos níveis pressóricos e da presença de acanthosis nigricans com o sexo das crianças participantes do estudo em Floriano-PI, 2015.

\begin{tabular}{|c|c|c|c|c|c|c|}
\hline \multirow[t]{2}{*}{ Variáveis } & \multicolumn{2}{|c|}{ Masculino } & \multicolumn{2}{|c|}{ Feminino } & \multirow[t]{2}{*}{ Total } & \multirow[t]{2}{*}{$P$} \\
\hline & $n=153$ & $\%$ & $n=196$ & $\%$ & & \\
\hline \multicolumn{7}{|l|}{ Estatura } \\
\hline$<140$ & 146 & $95,4 \%$ & 174 & $88,8 \%$ & 320 & 0,031 \\
\hline$>140$ & 7 & $4,6 \%$ & 22 & $11,2 \%$ & 29 & \\
\hline \multicolumn{7}{|l|}{ Peso } \\
\hline$<45$ & 148 & $96,7 \%$ & 190 & $96,9 \%$ & 338 & 0,913 \\
\hline$>45$ & 5 & $3,3 \%$ & 6 & $3,1 \%$ & 11 & \\
\hline \multicolumn{7}{|l|}{ Diagnóstico nutricional } \\
\hline Eutróficas & 115 & $75,2 \%$ & 133 & $67,9 \%$ & 248 & 0,154 \\
\hline Alteração nutricional ${ }^{*}$ & 38 & $24,8 \%$ & 63 & $32,1 \%$ & 101 & \\
\hline \multicolumn{7}{|c|}{ Percentil circunferência abdominal $\geq 90$} \\
\hline Sim & 12 & $3,4 \%$ & 14 & $4,0 \%$ & 26 & \\
\hline Não & 141 & $40,4 \%$ & 182 & $52,1 \%$ & 323 & 0,805 \\
\hline \multicolumn{7}{|c|}{ Classificação da pressão arterial } \\
\hline Normal & 139 & $90,8 \%$ & 186 & $94,9 \%$ & 325 & 0200 \\
\hline Hipertensão em estágio1 & 14 & $9,2 \%$ & 10 & $5,1 \%$ & 24 & $0, \angle 00$ \\
\hline \multicolumn{7}{|l|}{ Acanthosis nigricans } \\
\hline Não & 147 & $42,1 \%$ & 191 & $54,7 \%$ & 338 & 0,335 \\
\hline Sim & 6 & $1,7 \%$ & 5 & $1,4 \%$ & 11 & \\
\hline
\end{tabular}

* Inclui: Magreza acentuada; Magreza; Eutrofia; Sobrepeso; Obesidade; e Obesidade grave 
Tabela 4 - Associação da classificação da pressão arterial e da presença de acanthosis nigricans com o peso das crianças participantes do estudo em Floriano-PI, 2015. ( $n=349)$

\begin{tabular}{lcccccc}
\hline Variáveis & \multicolumn{2}{c}{$\begin{array}{l}\text { Peso (inferior a } \\
45 \mathrm{Kg} \text { ) }\end{array}$} & \multicolumn{2}{l}{$\begin{array}{l}\text { Peso (superior a } \\
45 \mathrm{Kg} \text { ) }\end{array}$} & Total & $P$ \\
& $\mathrm{n}=338$ & $\%$ & $\mathrm{n}=11$ & $\%$ & $\mathrm{n}=349$ & \\
\hline Classificação da pressão arterial & & & & & & \\
$\quad$ Normal & 318 & $94,1 \%$ & 7 & $63,6 \%$ & 325 & 0,004 \\
$\quad$ Hipertensão em estágio 1 & 20 & $5,9 \%$ & 4 & $36,4 \%$ & 24 & \\
Acanthosis nigricans I & 5 & $1,5 \%$ & 6 & $54,5 \%$ & 11 & 0,000 \\
$\quad$ Sim & 333 & $98,5 \%$ & 5 & $45,5 \%$ & 338 & \\
$\quad$ Não &
\end{tabular}

* Inclui: Magreza acentuada; Magreza; Eutrofia; Sobrepeso; Obesidade; e Obesidade grave

\section{DISCUSSÃO}

Embora existam alguns estudos sobre a prevalência da obesidade e HAS em crianças no Piauí, não foram encontradas pesquisas desenvolvidas na Cidade de Floriano-PI. No entanto, em uma investigação sobre a prevalência de excesso de peso infantil em escolas públicas da Cidade de Teresina-PI, capital do estado, com a participação de 230 escolares de sete a 10 anos, o sobrepeso chegou a atingir $20 \%$ do sexo masculino e $35 \%$ do sexo feminino, com a obesidade leve em $16 \%$ e $12 \%$ e obesidade grave em $6 \%$ e $3 \%$, respectivamente ${ }^{17}$. Percebe-se que a maior prevalência de alterações nutricionais no gênero feminino, em especial sobrepeso, foi consistente com os dados dessa pesquisa.

Em uma pesquisa que objetivou estabelecer a prevalência da HAS em crianças e adolescentes na faixa etária de seis a 17 anos, identificou-se, em uma amostra de 630 crianças e adolescentes, que $70,3 \%$ eram eutróficos, $3,9 \%$ estavam com baixo peso e $11,9 \%$ encontravam-se com obesidade, enquadrando-se em um grupo de risco e de atenção, dados que também se aproximam aos encontrados no estudo em tela ${ }^{18}$.

Foram identificados resultados diferentes em um exame clínico realizado com 2.980 crianças da região metropolitana de Santiago, Chile, o qual identificou prevalências de $6,7 \%$ de baixo peso, $8,9 \%$ de eutrofia, $13,6 \%$ de sobrepeso e $26 \%$ de obesidade. No Texas, Estados Unidos, uma investigação sobre a prevalência de excesso de peso em crianças, a adiposidade central apresentou a seguinte distribuição: $28,7 \%$ apresentavam sobrepeso, $46,5 \%$ eram eutróficas e 1,3\% apresentaram magreza ${ }^{19}$.

Ainda são altas as prevalências de sobrepeso e obesidade nas crianças mundialmente, mas nas duas últimas décadas, observa-se uma estabilização em alguns países, como Dinamarca e Groenlândia ${ }^{20}$. No Brasil estas estatísticas continuam ainda altas e são consideradas condições desafiadoras para os sistemas de saúde ${ }^{12}$. 
De acordo com os percentis de classificação da CA, apesar desta constituir um bom preditor de obesidade abdominal e para desenvolvimento de DCV, ainda existe uma deficiência de estudos quanto ao ponto de corte da CA em crianças. Um inquérito epidemiológico realizado no Texas, Estados Unidos, comprovou que o sobrepeso e a CA elevada aumentam 4,8 vezes mais as chances de ter PA elevada ${ }^{19}$.

Quanto à distribuição das crianças segundo verificações da PA com alteração dos percentis, verifica-se que a maioria das crianças desta investigação pode ser classificada como normotensa. No entanto, o número significativo de crianças apresentando valores limítrofes indica uma necessidade de acompanhamento em longo prazo para realmente determinar o quadro de HAS que a criança se enquadra. Apesar do estudo em tela não ter identificado elevadas prevalências para obesidade abdominal, o peso acima de $45 \mathrm{~kg}$ apresentou associação significativa com alterações na PA e presença de Acanthosis nigricans.

Um estudo com crianças e adolescentes de 7 a 17 anos, residentes em Erechim, Rio Grande do Sul, corrobora com os achados desta pesquisa, mostrou que $74,46 \%$ dos participantes avaliados apresentaram valores pressóricos abaixo do percentil 90, considerados normotensos e um contingente significativo de $12,53 \%$ com indicadores de PA considerada limítrofe. Classificados como hipertensos estágio 1 , obtiveram $7,14 \%$ e, como hipertensos em estágio 2 foi de $5,87 \%$, diferentemente do presente estudo que não apresentou crianças com esta classificação ${ }^{18}$.

Verifica-se que a maioria dos estudos mostrou que a HAS não está presente apenas em adultos, mas pode ser encontrada em crianças que já apresentam prevalência significativa para o desenvolvimento de DCV, como no norte da Califórnia, onde $22 \%$ das crianças avaliadas apresentaram préhipertensão ou HAS ${ }^{21}$.

Em uma pesquisa em crianças e adolescentes da Região do Caparaó-ES, foi analisada a PA em 1.342 crianças e adolescentes entre 7 a 14 anos. Realizouse a aferição dos níveis pressóricos em três momentos e observou-se que as prevalências eram diferenciadas para cada momento: a primeira aferição da PA indicou uma prevalência de níveis pressóricos elevados em $5,6 \%$, a segunda foi de $3,2 \%$ e a terceira chegou a $1,9 \%^{2}$. Por esse motivo, torna-se importante o acompanhamento das crianças desta pesquisa, pois é a forma mais fidedigna para realizar o diagnóstico da HAS.

Uma medida de aferição não é o suficiente para avaliar a PA de uma criança, pois existem diversos fatores que podem alterar os valores de PA. No entanto, enfatizase que todas as técnicas necessárias e estabelecidas para aferição de PA em crianças foram rigorosamente seguidas pela equipe responsável pela coleta e dados, certificando que, no momento da coleta de dados, os valores da PA das crianças avaliadas estavam, de fato, alterados.

Com relação aos achados clínicos relativos à Acanthosis nigricans, um estudo que tinha o objetivo de verificar as 
prevalências de obesidade, HAS, aumento de cintura abdominal e Acanthosis nigricans em estudantes de Sorocaba, São Paulo, verificou a presença desta alteração em um pequeno número de crianças $(3,8 \%)$ corroborando com a pesquisa em tela ${ }^{22}$.

A avaliação dos dados sobre os antecedentes familiares permitiu estabelecer que a investigação dos componentes genéticos é essencial para a investigação dos FRCV em crianças, podendo ser um dos marcadores mais importantes durante a anamnese clínica do paciente pediátrico.

Em uma pesquisa em Porto Alegre, Rio Grande do Sul, em que se analisou as variáveis obesidade, dislipidemia e diabetes mellitus em crianças e adolescentes, verificou-se que os antecedentes familiares apresentavam prevalências inferiores às encontradas no presente estudo. Os familiares de primeiro grau apresentavam 12,6\% para obesidade; $16,8 \%$ para dislipidemia e $6,7 \%$ para diabetes mellitus ${ }^{23}$.

Outro inquérito epidemiológico, desenvolvido em Curitiba com 410 crianças e adolescentes com idades entre 10 e 17 anos, que objetivou investigar a influência do IMC dos pais sobre o IMC dos seus filhos verificou que os índices de prevalência paterna de sobrepeso foram de $36 \%$ e de obesidade $13 \%$, enquanto que os índices maternos foram de $25 \%$ e $19 \%$, respectivamente. No que concerne ao risco relativo para obesidade, apresentou um risco em torno de 1,97 a 2,65 vezes maior para sobrepeso ou obesidade quando comparados com indivíduos filhos de pais eutróficos ${ }^{24}$.
Sendo assim é possível determinar que a hereditariedade é um dos FRCV responsável por predispor pais e filhos tanto para obesidade como para HAS e consequentemente as suas doenças associadas, dislipidemias, diabetes e distúrbios cardiovasculares que se manifestam em algum momento da vida. Destaca-se ainda que a influência genética e o modo de vida adotado no ambiente em que a criança vive é outro fator de risco importante para o surgimento de obesidade e HAS em formas precoces na infância ou em outra fase da vida, tornando desta forma essencial o rastreamento das DCV nas crianças através dos antecedentes e hábitos familiares.

Em relação à variável sobre a realização do AME e/ou complementar maior ou igual a quatro mês, identificou-se resultados semelhantes aos de um estudo analítico realizado na Austrália em que a maioria das crianças $(92,4 \%)$ havia sido amamentada e mais da metade $(54,2 \%)$ foi amamentada por seis meses ou mais. Neste estudo a maioria das crianças $(69,6 \%)$ estava eutróficas, $18,7 \%$ estavam acima do peso e mais $6,4 \%$ eram obesos ${ }^{25}$.

Pesquisadores realizaram um estudo com 125 crianças de crianças entre 5 a 10 anos em Lisboa e verificaram que $28,8 \%$ das participantes foram amamentadas por um período igual ou superior a seis meses e $25,6 \%$ não foram amamentadas. Desta forma observa-se que o risco de prevalência de sobrepeso e obesidade é maior nas crianças amamentadas menos de seis meses $(22,2 \%)$ quando comparativamente às amamentadas por seis meses ou mais $(0,8 \%)^{26}$. Observou-se 
que durante a busca de artigos em periódicos no Brasil são poucos os estudos que fazem a associação da variável tempo de aleitamento materno com o excesso de peso em crianças.

Aassociação entre osexoe odiagnóstico nutricional obteve resultados diferentes ao da pesquisa. Um estudo transversal na Cidade de Maceió, Alagoas, com 518 crianças de ambos os sexos, determinou que as crianças do gênero masculino apresentaram chance de obesidade e sobrepeso de $5,77 \%$ e $19,19 \%$, respectivamente, maior para o sobrepeso em comparação às crianças do gênero feminino 27. Outra pesquisa revela que o sobrepeso teve maior prevalência no sexo feminino $(19,9 \%)$, corroborando com o presente estudo ${ }^{23}$.

No que concerne à associação do sexo com CA, um estudo desenvolvido em Fortaleza, Ceará, verificou também que o sexo feminino apresentou maior prevalência para elevação da medida da CA $(27,5 \%)^{28}$.

Em outra investigação que realizou uma associação do gênero e PA, identificouse que na primeira aferição $6 \%$ dos meninos e $7 \%$ das meninas foram classificados como limítrofes, e que $3 \%$ das meninas e $4 \%$ dos meninos foram classificados com HAS em estágio 1. Resultados estes diferentes ao do presente estudo, que verificou que o gênero feminino apresentou maiores prevalências de
PA limítrofes e HAS em estágio 1, atingindo $7,4 \%$ e $2,8 \%$, respectivamente ${ }^{18}$.

\section{CONCLUSÃO}

Por meio deste estudo foi possível verificar um número significativo de crianças que se apresentou eutróficas e normotensas. Entretanto, uma porcentagem considerável exibiu sobrepeso e obesidade. A CA elevada e a presença de Acanthosis nigricans, assim como percentis de PA alterada no primeiro momento foram averiguadas em menor número, embora também se apresentem como um fato preocupante para a saúde pública da Cidade de Floriano-PI. A associação entre o peso elevado e PA alterada e presença de Acanthosis nigricans confirmam os achados da literatura científica e enfatizam a necessidade de abordagem conjunta dos fatores de risco cardiovascular, ainda na infância.

A partir dos resultados do presente estudo alerta-se para a necessidade de rastrear os fatores de risco cardiovascular na infância, possibilitando aos profissionais de saúde o desenvolvimento de ações e medidas para prevenção de DCV, especificamente a obesidade e HAS nas crianças. Faz-se necessário a implementação de políticas públicas na prevenção primária para minimizar os FRCV e um acompanhamento pelos profissionais de saúde. 


\section{REFERÊNCIAS}

1. Goulart, FAA. Doenças Crônicas Não Transmissíveis: Estratégias de Controle e Desafios e Para os Sistemas de Saúde. Brasília, DF: OPAS, OMS, Ministério da Saúde; 2011.

2. Ferreira CEF, Faria RJ, Bazoni PS. Hipertensão Arterial em Crianças e Adolescentes Moradores da Região do Caparaó, ES-Brasil. Rev Bras Cardiol.2013; 26(4):267271.

3. Sociedade Brasileira de Pediatria. Prevenção na infância das doenças do adulto: prevenção da doença cardiovascular. Rio de Janeiro: SBP; Departamento Científico de Nutrologia; 2006.

4. Crispim PAA, Peixoto MRG, Jardim PCB. Fatores de Risco Associados aos Níveis Pressóricos Elevados em Crianças de dois a cinco anos. Arq Bras Cardiol. 2014;102(1):39-46.

5. Coelho EF, Ferreira RE, Oliveira TFB, Navarro CA, Ferreira RM, Vianna JM et al. Prevalência de Fatores de Risco para Doença Cardiovascular em Trabalhadores de Empresa Siderúrgica. R Bras Ci Saúde. 2014; 189(Sup.4):1-58.

6. Bancalari R, Díaz C, Martínez-Aguayo A, Aglony M, Zamorano J, Cerda V et al. Prevalence of hypertension in school age children and its association with obesity. Revista Medica de Chile. 2011; 139(7):872-879.

7. Gupta N, Goel K, Shah P, Misra A .Childhood obesity in developing countries: Epidemiology, Determinants, and Prevention. Endocr Rev. 2012; 33(1):48-78.

8. Moyer VA. Screening for Primary Hypertension in Children and Adolescents: U.S. Preventive Services Task Force Recommendation Statement. Annals of Internal Medicine. 2013; 159(9):613-619.

9. Sociedade Brasileira de Hipertensão. Doença de adulto, 2014. Disponível em: http://www.sbh.org.br/geral/sbh-namidia. asp?id=254. Acesso em: 19 de setembro de 2014

10. Pires ACL, Moreira PVL. Aspectos Antropométricos, Nutricionais e de Atividade Física em Escolares do Bairro do Grotão, João Pessoa/PB. R Bras Ci Saúde. 2011;15(3): 339-348.

11. Hockenberry MJ, Wilson, D. Wong, Fundamentos de enfermagem pediátrica. Tradução Maria Inês Corrêa Nascimento. 9a. ed. Rio de Janeiro: Elsevier; 2014.

12. Brasil. Ministério da Saúde. Secretaria de Atenção à Saúde. Departamento de Atenção Básica. Saúde na escola. Brasília, DF: Ministério da Saúde;2009. (Série B. Textos Básicos de Saúde) (Cadernos de Atenção Básica; n. 24)

13. Sociedade Brasileira De Pediatria. Avaliação nutricional da criança e do adolescente - Manual de Orientação / Sociedade Brasileira de Pediatria. Departamento de Nutrologia. São Paulo: Sociedade Brasileira de Pediatria. Departamento de Nutrologia; 2009.
14. Andrade JP, Nobre F. Sociedade Brasileira de Cardiologia. Sociedade Brasileira de Hipertensão. Sociedade Brasileira de Nefrologia. VI Diretrizes Brasileiras de Hipertensão. Arq Bras Cardiol. 2010; 95(Supl.1):1-51.

15. Brasil. Ministério da Saúde. Resolução n. 466, de 12 de dezembro de 2012. Aprovar as seguintes diretrizes e normas regulamentadoras de pesquisas envolvendo seres humanos. Decreto de Delegação de Competência. Brasília. Diário Oficial [da] Republica Federativa do Brasil, Brasília, DF, n. 12, 13 junho de 2013. Seção 1, p. 59.

16. Nhanhes III, Center for disease control and prevention. The third national health and nutrition examination survey (Nhanhes III, 1988-1994). References and manual report. Bethesda, Md: National center for health statistics; 2005.

17. Ribeiro GM, Silva LML, Ibiapina DFN. Prevalência de excesso de peso infantil em escolas públicas de Teresina - PI. R. Interd. 2014; 79(1):162-166.

18. Denti IA, Selivon G, Serpa MA. Prevalência da hipertensão arterial sistêmica em crianças e adolescentes. Perspectiva. 2012; 36(133): 31-39.

19. Meininger, Janet C. et al. Overweight and Central Adiposity in School-Age Children and Links with Hypertension. Pediatr Nurs. 2011; 25(2):119-125.

20. Wabitsch M, moss A, Hauschild KK. Unexpected plateauing of childhood obesity rates in developed countries. BMC Public Health, 2014; 12(17): 1-5, 2014.

21. Frigaard M, Goto k, Wolff $C$, Bianco-Simeral S, Fahey $T$. Identifying Red Flags: Using Anthropometry Measures to Screen for Elevated Blood Press. ICAN: Infant, Child, \& Adolescent Nutrition. 2013; 5(1):37-41.

22. Mazaro IAR, Zanolli ML, Antonio ARGM, Morcilo AM, Zambon MP. Obesidade e fatores de risco cardiovascular em estudantes de Sorocaba, SP. Rev. Assoc. Med. Bras.2011; 57(6): 674-680.

23. Schommer VA, Barbiero SM, Cesa CC, Oliveira R, Silva AD, Pellanda LC. Excesso de peso, variáveis antropométricas e pressão arterial em escolares de $10 \mathrm{a}$ 18 anos. Arq Bras Cardiol. 2014; 102(4):312-318.

24. Mascarenhas LPG, Amer NM, Bouszewski MCS, Lacerdo Filho L, Prati FS. Influência do excesso de peso dos pais em relação ao sobrepeso e obesidade dos filhos. Pensar a Prática. 2013; 16(2):320-618.

25. Scott, JA, Ng SY, Cobiac L. The relationship between breastfeeding and weight status in a national sample of Australian children and adolescentes. BMC Public Health. 2012; 12(107):1-6.

26. Ferraria N, Rodrigues V, Macedo L. Aleitamento materno e excesso de peso em crianças na idade escolar. Sci. Med. 2013; 23(2):75-81. 
27. Silva $Y M Q$, Kraguljac $M$, Albuquerque RB, Fonseca FS. Prevalência de excesso de peso em crianças e adolescentes de um projeto esportivo em Maceió-AL. R Bras Ci Saúde. 2014;18(Sup.4):67-74.

28. Macêdo SF, Araújo MFM, Marinho PB, Lima ACS, Freitas RWF, Damasceno MMC. Fatores de risco para diabetes mellitus tipo 2 em crianças. Rev. Latino-Am. Enfermagem. 2010; 18,(5):1-8.

\section{CORRESPONDÊNCIA}

Alaeny Dias Clementino da Silva

Rua João Viana de Carvalho, 224, Bairro Sambaíba Velha, Floriano, Brasil.

CEP: 64803-242.

Email: alaeny_dias@hotmail.com 